\title{
Impacto de una intervención alimentaria con un concentrado proteico de Medicago sativa $L$ (alfalfa), en niños pre escolares con desnutrición crónica
}

\author{
Nourishing intervention impact with Medicago sativa $L$ (alfalfa) protein extract in chronically \\ undernourished preschool children
}

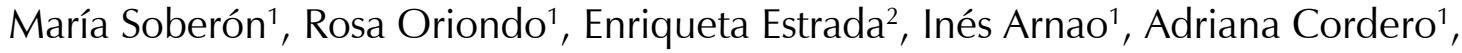 \\ Luz Velásquez ${ }^{1}$, Irene Arteaga ${ }^{2}$ \\ ${ }^{1}$ Centro de Investigación de Bioquímica y Nutrición, Facultad de Medicina, Universidad Nacional Mayor de San Marcos. Lima, Perú. \\ ${ }^{2}$ Escuela Académico Profesional de Nutrición Humana, Facultad de Medicina, Universidad Nacional Mayor de San Marcos. Lima, Perú.
}

\begin{abstract}
Resumen
Introducción: Estudios realizados a nivel preclínico han demostrado el valor biológico del concentrado proteico obtenido de Medicago sativa L, alfalfa (CPA), por lo que este producto puede ser una alternativa para reducir la desnutrición. Objetivo: Evaluar el efecto del consumo de CPA por niños preescolares con desnutrición crónica, mediante indicadores bioquímicos y hematológicos. Diseño: Estudio analítico de intervención, de tipo longitudinal y prospectivo. Institución: Centro de Investigación de Bioquímica y Nutrición, Facultad de Medicina, Universidad Nacional Mayor de San Marcos. Participantes: Niños preescolares con desnutrición crónica, provenientes de una comunidad de Pampas, Huancavelica, Perú. Intervenciones: Previo consentimiento informado de los padres, se evaluó el estado alimentario-nutricional, bioquímico y hematológico de 23 niños menores de seis años, para establecer la condición de desnutrición. Luego, se desarrolló una intervención alimentario nutricional con el CPA como complemento alimenticio, por 25 días, en niños seleccionados. Finalmente, se repitió la evaluación una vez concluido el período de intervención. Las determinaciones bioquímicas fueron hemoglobina, proteínas totales, albúmina y globulina, creatinina, transaminasas, y las hematológicas, hematocrito, recuento de reticulocitos. Se utilizó el programa Excel y t-student, a fin de obtener la inferencia en los resultados. Resultados: Para el indicador talla/edad, 84,5\% de los niños padecía desnutrición crónica; respecto a calorías y nutrientes consumidos por los niños, los valores de energía estuvieron entre 563 kcal y 2597 kcal; de proteína, 9,7 g a 78 g; de grasas, 5,1 g a 53,8 g; carbohidratos, 79,8 g a 495,1 g; calcio, entre 180,9 mg y 986 mg; y hierro, 1,9 mg y 21,5 mg. Los valores de hemoglobina, proteínas totales, globulinas, creatinina, transaminasas y hematocrito obtenidos antes y después de la ingesta de CPA, no presentaron variaciones $(p>0,05)$; pero, los valores de albúmina y recuento de reticulocitos se incrementaron significativamente después de la ingesta $(p<0,05)$. Conclusiones: El consumo diario de CPA por niños con desnutrición crónica por 25 días aumentó significativamente los valores de recuento de reticulocitos, así como los niveles de albúmina sérica.
\end{abstract}

Palabras clave: Medicago sativa; desnutrición proteica; albúminas; reticulocitos.

\begin{abstract}
Introduction: Studies made at preclinical level have demonstrated the Medicago sativa $L$, alfalfa (CPA)'s concentrated protein biological value; as such this product could be an alternative to reduce undernourishment. Objective: To determine CPA consuming effects in kindergarten children with chronic undernourishment by biochemical and hematologic indicators. Design: Intervention analytical, longitudinal and prospective study. Setting: Biochemistry and Nutrition Research Center, Faculty of Medicine, Universidad Nacional Mayor de San Marcos. Participants: Pampas, Huancavelica, Peru, preschool children with chronic undernourishment. Interventions: Previous parents informed consent we determined the nourishment-nutritional, biochemical and hematologic status of 23 less than six year-old children in order to establish undernourishment. Then a CPA nutritional nourishment intervention in the chronically undernourished children was developed for 25 days and finally the initial evaluation was repeated. Biochemical -hemoglobin, total proteins, albumin and globulin, creatinine, transaminases- and hematologic -hematocrit, reticulocytes count- determinations were done before and after providing the CPA nutritional complement. Excel program and t-student were used in order to obtain results inference. Results: By stature/age indicator $84,5 \%$ of the children suffered chronic undernourishment. With respect to calorie and nutrients consumed by the children, energy values were between $563 \mathrm{kcal}$ and 2597 kcal, protein $9,7 \mathrm{~g}$ to $78 \mathrm{~g}$, lipids $5,1 \mathrm{~g}$ to $53,8 \mathrm{~g}$, carbohydrates from $79,8 \mathrm{~g}$ to $495,1 \mathrm{~g}$, calcium between $180,9 \mathrm{mg}$ and $986 \mathrm{mg}$, and iron from $1,9 \mathrm{mg}$ to $21,5 \mathrm{mg}$. Hemoglobin, total proteins, globulins, creatinine, transaminases and hematocrit before and after CPA ingestion did not display variations $(p>0,05)$ but albumin and reticulocytes count increased significantly following ingestion $(p<0,05)$. Conclusions: CPA daily consumption for 25 days CPA by children with chronic undernourishment significantly increased reticulocytes count and albumin levels.
\end{abstract}

Key words: Medicago sativa; protein malnutrition; albumins; reticulocytes.

\section{INTRODUCCIÓN}

El estado nutricional es el resultado de una amplia gama de condiciones sociales y económicas y constituye un indicador muy sensible del nivel general de desarrollo de un país. Si bien es cierto que durante los últimos 25 años en el Perú se han producido mejoras relativas en algunos factores relacionados con la nutrición, su grado de avance e inestabilidad aún condiciona y determina altas cifras de desnutrición, particularmente en las niñas y niños de las zonas más pobres del país.

En el Perú, según ENDES $2000^{(1)}$, la desnutrición crónica en menores de cinco años en zonas rurales se sitúa en 40,2\%. Estos índices varían considerablemente en la costa, sierra y selva, según niveles de pobreza y según área de residencia, siendo más altos en la sierra y selva, en familias pobres y en extrema pobreza y, además, en áreas rurales.
Uno de los departamentos de la sierra peruana más afectados es Huancavelica, donde la mitad de la población menor de cinco años está afectada por la desnutrición crónica $(53,4 \%)$, siendo $52 \%$ de niños de las áreas rurales y 36\% de las zonas urbanas, uno de los porcentajes más altos del país ${ }^{(2)}$. Este departamento tiene como segunda producción agrícola más importante el cultivo de alfalfa. Para el año 2002, la producción 
de alfalfa en Huancavelica fue 125602 TM, únicamente usada como forraje para animales ${ }^{(3)}$.

La alfalfa, Medicago sativa $L$, es una leguminosa altamente ecológica, de fácil cultivo y comercialización En el país, se cultiva como forraje para animales; sin embargo, nuestra población de escasos recursos utiliza de manera esporádica y empírica el jugo extraído de las hojas frescas de alfalfa como una alternativa para disminuir la anemia, combatir la tuberculosis, en niños, gestantes y adultos. En otros países, se ha elaborado alimentos que tienen como ingrediente un extracto de hojas de alfalfa, rico en macronutrientes (proteínas, azúcares y lípidos) y micronutrientes (vitaminas y oligoelementos) ${ }^{(4-6)}$.

En el Centro de Investigación de Bioquímica y Nutrición, de la Universidad Nacional Mayor de San Marcos (UNMSM), Lima, Perú (2005 a 2007) se ha realizado estudios sobre el valor biológico de un concentrado proteico obtenido de alfalfa (CPA), en ratas sometidas previamente a desnutrición crónica, encontrándose que este producto administrado como complemento con otras fuentes proteicas (caseína de la leche, proteína de kiwicha) mejora los niveles proteicos de estos animales de experimentación. CPA es también rico en hierro y fue asimilado por animales previamente sometidos a desnutrición sin originar toxicidad hepática o esplénica, durante el tiempo de consumo ${ }^{(7,8)}$, por lo que este producto se presenta como una alternativa para reducir la desnutrición.

Con el propósito de colaborar en la política de reducción de la desnutrición crónica infantil en zonas de riesgo alto asumida por el Estado y siendo Huancavelica una región de extrema pobreza, con una grave crisis socioeconómica, tasa alta de desnutrición infantil y, además, productor de alfalfa, el presente trabajo tuvo por objetivo evaluar bioquímica y hematológicamente el efecto del consumo de un concentrado proteico de alfalfa (CPA) en niños preescolares con desnutrición crónica, de la comunidad de Daniel Hernández, Pampas, Huancavelica, Lima, Perú.

\section{MÉTODOS}

Se realizó un estudio analítico de intervención, de tipo longitudinal y prospectivo. El estado nutricional fue determinado por mediciones antropométricas (edad, peso y talla) y encuestas alimentarionutricionales (encuestas de recordatorio de 24 horas, frecuencia de consumo de alimentos, hábitos y costumbres alimentarias), para determinar la cantidad de energía y de proteínas que consumía cada niño y luego relacionarlo con su requerimiento de acuerdo a su edad. Con estos datos, se pudo realizar el diagnóstico nutricional de 23 niños de ambos sexos, entre las edades de 4 y 5 años de edad, del centro de educación inicial № 460, La Colpa, del distrito de Daniel Hernández, Pampas, Huancavelica, ubicado a 3163 msnm.

A los padres de familia de dicho centro educativo se les explicó los objetivos y alcances del estudio a realizar, y a aquellos que aceptaron participar se les pidió firmar un consentimiento informado aprobado por el comité de Ética de la Facultad de Medicina Humana, de la Universidad Nacional Mayor de San Marcos, Lima, Perú.

Luego, se procedió a la toma de muestra sanguínea, de donde se obtuvo el suero y plasma respectivos y se procedió a almacenarlos en una cadena de frío, con el cuidado debido para su transporte al laboratorio del Centro de Investigación de Bioquímica y Nutrición, Facultad de Medicina, UNMSM, donde se realizó las determinaciones bioquímicas y hematológicas. Las determinaciones bioquímicas fueron: para hemoglobina, el método colorimétrico de cianmetahemoglobina (Valtex diagnostics); para proteínas totales, albúmina y globulinas, el método de biuret y verde de bromocresol, respectivamente (Audit diagnostics); para creatinina, el método de Jaffé, (Audit diagnostics) y transaminasas, por un método cinético (Valtex diagnostics). Las pruebas hematológicas realizadas fueron hematocrito y recuento de reticulocitos, según la metodología convencional llevada a cabo en los laboratorios clínicos.

Una vez obtenidos los datos anteriormente citados, se procedió a suministrar a los niños un concentrado proteico de alfalfa (CPA, en proceso para patente), en una cantidad de $10 \mathrm{mg}$ diarios, como alimento complementario al refrigerio, en la forma de sachet. La ingesta de CPA se realizó durante el tiempo que permanecieron los niños en el centro educativo, por 25 días. El CPA fue consumido por los niños sin ninguna dificultad. Al final del período de intervención alimentaria, se procedió nuevamente a evaluar los niños nutricional, bioquímica y hematológicamente.

Los datos antropométricos fueron procesados mediante programa Excel, diagnóstico de Waterloo. Para los datos bioquímicos obtenidos, se aplicó estadística descriptiva, es decir, promedios aritméticos, desviación estándar, $\mathrm{t}$ student, a fin de obtener la inferencia en los resultados obtenidos.

\section{RESULTADOS}

De los 23 niños evaluados, para el indicador peso/talla 4,8\% de ellos tenía desnutrición aguda; igual porcentaje se encontró niños en riesgo a desnutrirse; $19 \%$ y $22,6 \%$ de ellos presentaban sobrepeso y obesidad, respectivamente; $48,8 \%$ de los niños evaluados para ese indicador se encontraban dentro de los rangos normales (figura 1).

En relación al indicador talla/edad, encontramos que $84,5 \%$ de los niños tenía desnutrición crónica, observándose que los varones tenían mayor retardo en crecimiento $(51,2 \%)$ que las mujeres $(33,3 \%)$. Así mismo, 8,3\% se encontraba en riesgo de retardo de crecimiento (figura 2).

Con respecto al consumo energético, el mínimo fue $563 \mathrm{kcal}$ y el máximo 2597 kcal; y con relación al consumo de macro y micronutrientes, se pudo observar valores muy bajos y muy altos; los rangos de proteínas fueron de $9,7 \mathrm{~g}$ a $78 \mathrm{~g}$, las grasas entre $5,1 \mathrm{~g}$ y $53,8 \mathrm{~g}$ y los carbohidratos, entre $79,8 \mathrm{~g}$ y $495,1 \mathrm{~g}$. En cuanto a minerales como el calcio, los rangos estuvieron entre 180,9 mg y 986 $\mathrm{mg}$; y con respecto al hierro, entre $1,9 \mathrm{mg}$ y $21,5 \mathrm{mg}$ (tabla 1 ).

El porcentaje de adecuación de la dieta consumida por los niños para la energía 


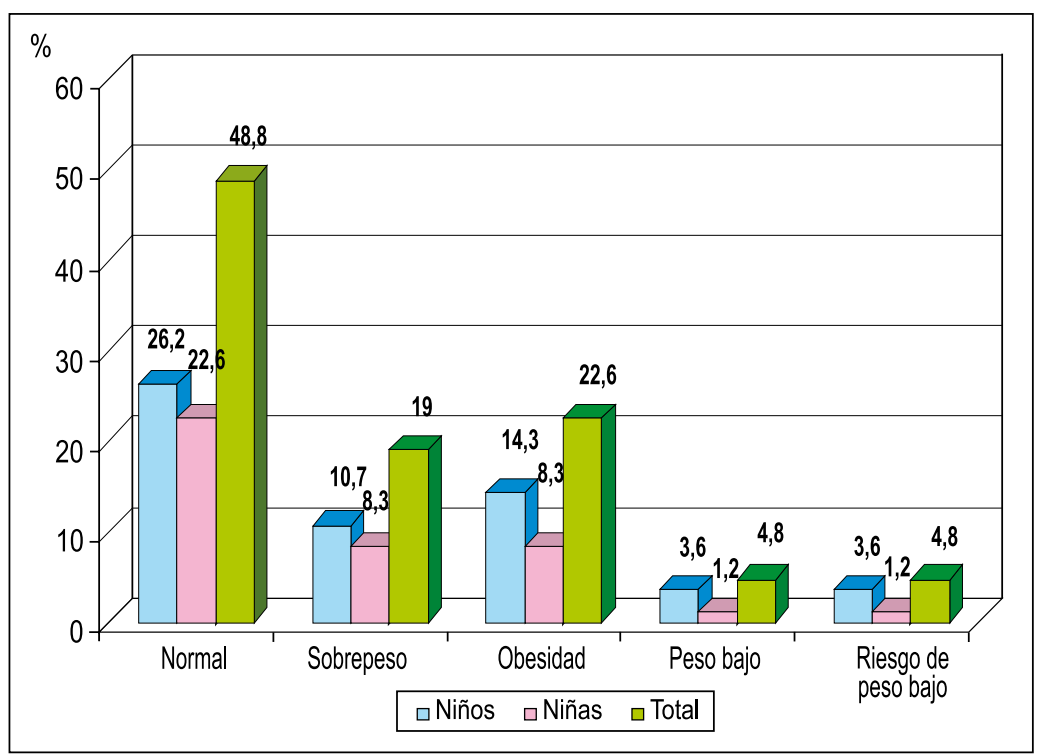

Figura 1. Estado nutricional de niños menores de 5 años, según el indicador peso/talla.

fue $90 \%$, para proteínas $87 \%$, para grasa $70 \%$, para calcio $56 \%$ y para hierro no hemínico 92\% (tabla 2).

Es conocido que los valores bioquímicos y hematológicos obtenidos en residentes a nivel del mar difieren de los de altura ${ }^{(9)}$, contándose por ello con valores referenciales para este último grupo. Al realizar la evaluación bioquímica y hematológica después de la intervención alimentaria, tres de los niños seleccionados fueron retirados por sus madres por lo que solo se obtuvo los datos completos de 20 niños. Al comparar los valores bioquímicos y hematológicos de esos 20 niños antes de la ingesta de CPA, observamos que estos datos se encuentran muy por debajo de los valores referenciales, lo que corrobora su estado de desnutrición (tabla 3 ).

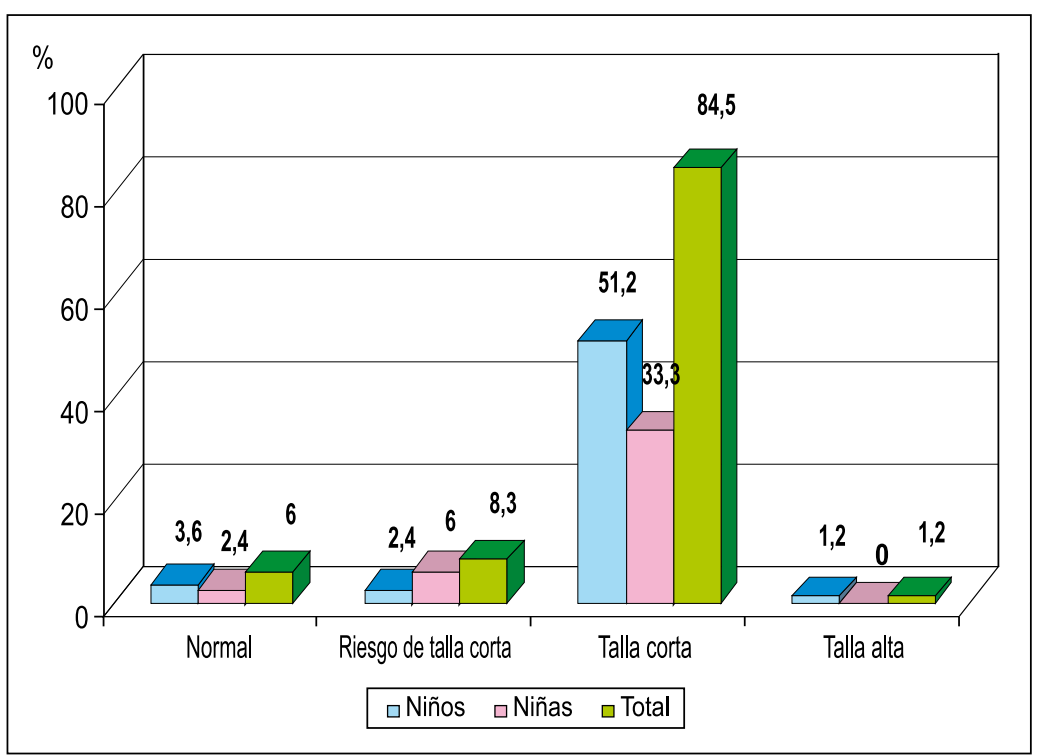

Figura 2. Estado nutricional de niños menores de 5 años, según el indicador talla/edad.
Los valores de hemoglobina, proteínas totales, globulinas, creatinina y transaminasas así como de hematocrito obtenidos antes de la ingesta (valores basales) y después de la ingesta, no presentaron variaciones significativas $(p>0,05)$. Pero, cuando se comparó los valores de albúmina (figura 3) y recuento de reticulocitos (figura 4), antes y después de la ingesta de CPA, el análisis estadístico dio un valor de $p<0,05$, siendo los valores de recuento de reticulocitos los que se incrementaron considerablemente en cada uno de los niños.

Los valores promedios obtenidos después de la ingesta de CPA se muestra en la tabla 4 .

Los resultados de talla y peso después de 25 días de ingesta no sufrieron variación.

\section{DISCUSIÓN}

La desnutrición proteico-calórica es uno de los problemas que más afectan a la población de menores de cinco años en los países en desarrollo. Para determinar su incidencia, se ha utilizado hasta hoy varios indicadores: clínicos, antropométricos, dietéticos, bioquímicos y estadísticas vitales. En salud pública, los indicadores más utilizados han sido los antropométricos y de estos la relación talla/edad y la relación peso/talla. La interpretación e interrelación de estos indicadores utilizados simultáneamente para evaluar el estado nutricional es de importancia en salud pública, ya que los criterios de selección para admisión de niños en programas de recuperación nutricional todavía se efectúan considerando estos parámetros

Por otro lado, el uso de los índices talla/edad y peso/talla en estudios de campo de prevalencia de malnutrición, puede tener la ventaja de dar una idea más clara del tipo de desnutrición prevalente y, además, es de valor para establecer prioridades de intervención nutricional. Además, se ha demostrado mediante el análisis de correlación y regresión múltiple que peso para la talla y talla para la edad son virtualmente independientes.

Considerando estas observaciones, el presente estudio realizó una evaluación 
Tabla 1. Consumo diario de calorías y nutrientes.

\begin{tabular}{lrrrrr}
\hline Nutrientes & Mínimo & Máximo & Mediana & P25 & P75 \\
\hline Energía kcal & 563,0 & 2597,0 & 1217,0 & 1008,0 & 1507,0 \\
Proteína g & 9,7 & 78,5 & 44,3 & 34,3 & 50,9 \\
Grasa g & 5,1 & 53,8 & 21,2 & 17,2 & 30,3 \\
Carbohidratos g & 79,8 & 495,1 & 203,2 & 183,9 & 264,8 \\
Fibra g & 1,0 & 21,0 & 4,9 & 3,6 & 8,0 \\
Calcio mg & 180,9 & 986,0 & 446,1 & 338,3 & 463,9 \\
Fósforo mg & 115,7 & 1906,9 & 811,1 & 693,6 & 971,9 \\
Hierro mg & 1,9 & 21,5 & 9,2 & 6,8 & 12,4 \\
Vitamina A $\mu$ g & 77,5 & 424,9 & 192,1 & 138,2 & 349,9 \\
Tiamina mg & 0,2 & 2,2 & 1,3 & 1,0 & 1,5 \\
Riboflavina mg & 0,5 & 2,8 & 1,2 & 1,0 & 1,4 \\
Niacina mg & 1,8 & 23,0 & 9,7 & 8,5 & 12,4 \\
Vitamina C mg & 5,9 & 150,0 & 62,7 & 45,7 & 83,9 \\
\hline
\end{tabular}

nutricional considerando los indicadores peso/edad y peso/talla. El indicador peso/talla reportó que 4,8\% de los niños y niñas tenía desnutrición aguda, cifra mucho más alta que lo encontrado a nivel nacional (2). Otro aspecto importante fue la presencia de niños con sobrepeso $(19 \%)$ y obesidad $(22,6 \%)$. a esta corta edad, resultados que son similares a lo encontrado por Pajuelo y colaboradores ${ }^{(10)}$. Esto es consecuencia de una alimentación rica en carbohidratos, deficiente en proteínas y en micronutrientes, que son importantes para el crecimiento y desarrollo de los mismos.

En relación al indicador talla/edad, podemos señalar que $8,3 \%$ de los niños se encontraba en riesgo de retardo de crecimiento, comparado con los estándares a nivel departamental (43\%) y nacional
$(25,4 \%)$. Estas cifras son elevadas en relación a lo encontrado a nivel nacional (2), lo que podría indicar que existen factores importantes que inciden en el normal crecimiento y desarrollo de estos niños. Entre las causales más importantes referidas en la literatura se puede comentar a los hábitos alimentarios inadecuados en la época de ablactancia y el consumo de alimentos inadecuados por desconocimiento del valor nutricional de los mismos, por parte de las madres. Tanto la calidad como la cantidad inadecuada de consumo de alimentos son determinantes en la cobertura de sus requerimientos nutricionales. En tal sentido, la educación alimentaria nutricional y la provisión de recursos y/o suplementos nutricionales podrían coadyuvar al mejoramiento de la salud y nutrición de los niños y niñas,
Tabla 2. Porcentaje de adecuación de la dieta consumida.

\begin{tabular}{lccc}
\hline Energía y nutrientes & Recomendados & $\begin{array}{c}\text { Consumo } \\
\text { mediana }\end{array}$ & $\begin{array}{c}\text { Porcentaje } \\
\text { adecuación }\end{array}$ \\
\hline Energía (kcal) & $1350^{*}$ & 1217 & 90 \\
Proteína (g) & $51^{*}$ & 44 & 87 \\
Grasa $(\mathrm{g})$ & $30^{*}$ & 21 & 70 \\
Carbohidratos $(\mathrm{g})$ & $219^{*}$ & 203 & 93 \\
Calcio $(\mathrm{mg})$ & $800^{*}$ & 446 & 56 \\
Hierro $(\mathrm{mg})$ & $10^{* *}$ & 9 & 92 \\
Vitamina A $(\mu \mathrm{g})$ & $400^{* *}$ & 192 & 48 \\
Tiamina $(\mathrm{mg})$ & $0,6^{* *}$ & 1,3 & 104 \\
Riboflavina $(\mathrm{mg})$ & $0,6^{* *}$ & 1,2 & 200 \\
Niacina $(\mathrm{mg})$ & $8^{* *}$ & 9,7 & 121 \\
Vitamina C $(\mathrm{mg})$ & $25^{* *}$ & 63 & 252 \\
\hline
\end{tabular}

* Cereceda MP. Dietética de la teoría a la práctica. Lima: Ed. UNMSM; $2008{ }^{(11) .}$

** FAO/OMS. Ingesta dietética recomendada. permitiendo revertir el problema nutricional a corto plazo ${ }^{(11)}$.

Estudios realizados en países desarrollados y en vías de desarrollo demuestran que la deficiencia de hierro es la principal causa de anemia en mujeres y que el suplemento con hierro previene o corrige este déficit ${ }^{(12,13)}$. Si bien la anemia per se tiene un impacto en la salud, la deficiencia misma de hierro también lo es. Por ejemplo, en niños de ocho meses de edad con hemoglobina $<9,5 \mathrm{~g} / \mathrm{dL}$ se observa daño en el desarrollo motor ${ }^{(14)}$. De ahí la importancia de identificar la deficiencia de hierro, particularmente, en este segmento vulnerable de la población. Además, la principal diferencia entre el metabolismo del niño y del adulto está dada por la dependencia que tienen los primeros del hierro proveniente de los alimentos. En los adultos, aproximadamente 95\% del hierro necesario para la síntesis de la hemoglobina proviene de la recirculación del hierro de los hematíes destruidos. En contraste, un niño entre los 4 y 12 meses de edad utiliza 30\% del hierro contenido en los alimentos con este fin, y la tasa de reutilización a esta edad es menos significativa ${ }^{(15)}$.

La medición de hemoglobina y hematocrito se relaciona indirectamente con la concentración de hierro. Por tal motivo, estos parámetros fueron evaluados en los niños seleccionados para el presente estudio, encontrándose que después de 25 días de haber ingerido el CPA no hubo un aumento significativo en dichos parámetros, debido al corto tiempo de la intervención alimentaria y del espacio de tiempo entre la primera y segunda toma de sangre y $(\mathrm{Hb}=13,39$ y 13,$48 ;$ Hto $=$ $38,4$ y 38,6$)$.

En la altura, los valores de hemoglobina varían con respecto a los valores obtenidos a nivel del mar. González y colaboradores comunicaron una condición de anemia para valores de hemoglobina de 12,9 a $13,7 \mathrm{~g} / \mathrm{dL}$ y de hematocrito, $39 \%$ a $41,5 \%$, en madres gestantes peruanas que residen entre 3000 y $3500 \mathrm{~m}$ de altitud, comparados con los valores de $11 \mathrm{~g} / \mathrm{dL}$ y $33 \%$, respectivamente, obtenidos a nivel del mar ${ }^{(16)}$. En niños de 5 a 11 años de edad que residen a nivel del mar, valores de hemoglobina inferiores a $11,5 \mathrm{~g} / \mathrm{dL}$ y de 
Tabla 3. Valores promedios de la evaluación bioquímica y hematológica obtenido en los niños seleccionados, antes de la ingesta de concentrado proteico obtenido de alfalfa (CPA).

\begin{tabular}{lcccccc}
\hline & $\begin{array}{c}\text { Hemoglobina } \\
(\mathrm{g} / \mathrm{dL})\end{array}$ & $\begin{array}{c}\text { Hematocrito } \\
(\%)\end{array}$ & $\begin{array}{c}\text { Recuento de } \\
\text { reticulocitos } \\
(\%)\end{array}$ & $\begin{array}{c}\text { Proteínas } \\
\text { totales } \\
(\mathrm{g} / \mathrm{dL})\end{array}$ & $\begin{array}{c}\text { Albúmina } \\
(\mathrm{g} / \mathrm{dL})\end{array}$ & $\begin{array}{c}\text { Globulina } \\
(\mathrm{g} / \mathrm{dL})\end{array}$ \\
\hline Media & 13,39 & 38,4 & 1,54 & 7,19 & 3,85 & 3,33 \\
$\mathrm{DE}$ & 0,589 & 1,68 & 0,71 & 0,67 & 0,28 & 0,76 \\
$\mathrm{RR}^{*}$ & 15 a 18 & $\mathrm{NR}$ & $2 \mathrm{a} 5$ & 7,5 a 9,0 & $4,5 \mathrm{a} 6,5$ & $\mathrm{NR}$ \\
\hline
\end{tabular}

$\mathrm{DE}=$ Desviación estándar.

$\mathrm{RR}^{*}=$ Rango referencial en niños de 2 a 6 años que habitaban en lugares por encima de $3000 \mathrm{msnm}$, utilizados por el Laboratorio de Bioquímica y Hematología de la Maternidad de Lima.

$\mathrm{NR}=$ No reportado.

hematocrito por debajo de $34 \%$ son puntos de referencia para considerar un estado de anemia. Sin embargo, estos valores referenciales no son aplicables a niños que viven por encima de los 3000 metros de altitud. Existen estudios realizados, hace más de diez años, que comunican que los valores del hematocrito en niños nativos de la altura (3 400 y $4341 \mathrm{msnm}$ ) son mayores que los de adultos a nivel del mar ${ }^{(17,18)}$, lo cual hace evidente el efecto de la altura sobre la eritropoyesis. Esto se evidencia nuevamente en los niños seleccionados para este estudio, cuyos valores de hemoglobina y hematocrito estuvieron muy por encima de los valores referenciales a nivel del mar $(13,39 \mathrm{~g} / \mathrm{dL}$ y $38,4 \%$, respectivamente); sin embargo, presentaban indicadores antropométricos -como el indicador talla/edad-y nutricionales -como el consumo de hierro dietario y proteínas- que los clasificaban en una condición de desnutrición y anemia.

El recuento reticulocitario permite medir el índice de eritropoyesis y evaluar la respuesta de la médula ósea ante una anemia, o para vigilar el tratamiento de la anemia. Esto permitió evaluar la respuesta de la médula ósea al tratamiento con CPA. Por otro lado, los valores referenciales que se maneja en un laboratorio clínico se aplican tanto para un recién nacido como para niños menores de 6 años. No se ha encontrado estudios que muestren diferencias significativas de los valores de recuento de eritrocitos entre niños menores de 6 años residentes a nivel de mar con aquellos que residen por encima de los $3000 \mathrm{~m}$ de altitud. Lo que se puede observar en los resultados obtenidos con CPA es un incremento significativo de estos valores después de 25 días de consumo $(p<0,05)$ (figura 4$)$, debido probablemente a una buena absorción de los nutrientes presentes en el CPA. Esto se debería a que la anemia presente en estos niños es de tipo ferropénica y que puede ser revertida con una ingesta adecuada de proteínas, micronutrientes como hierro, entre otros, y el consumo de CPA puede proporcionarlo.

La albúmina es la proteína más abundante de la sangre, sintetizada por el hígado, que cumple importantes funciones en el transporte de sustancias endógenas y exógenas; además, es usada como un indicador del estado nutricional, por su función de reserva de aminoácidos y su corto tiempo de recambio. De los marcadores bioquímicos estudiados, son los

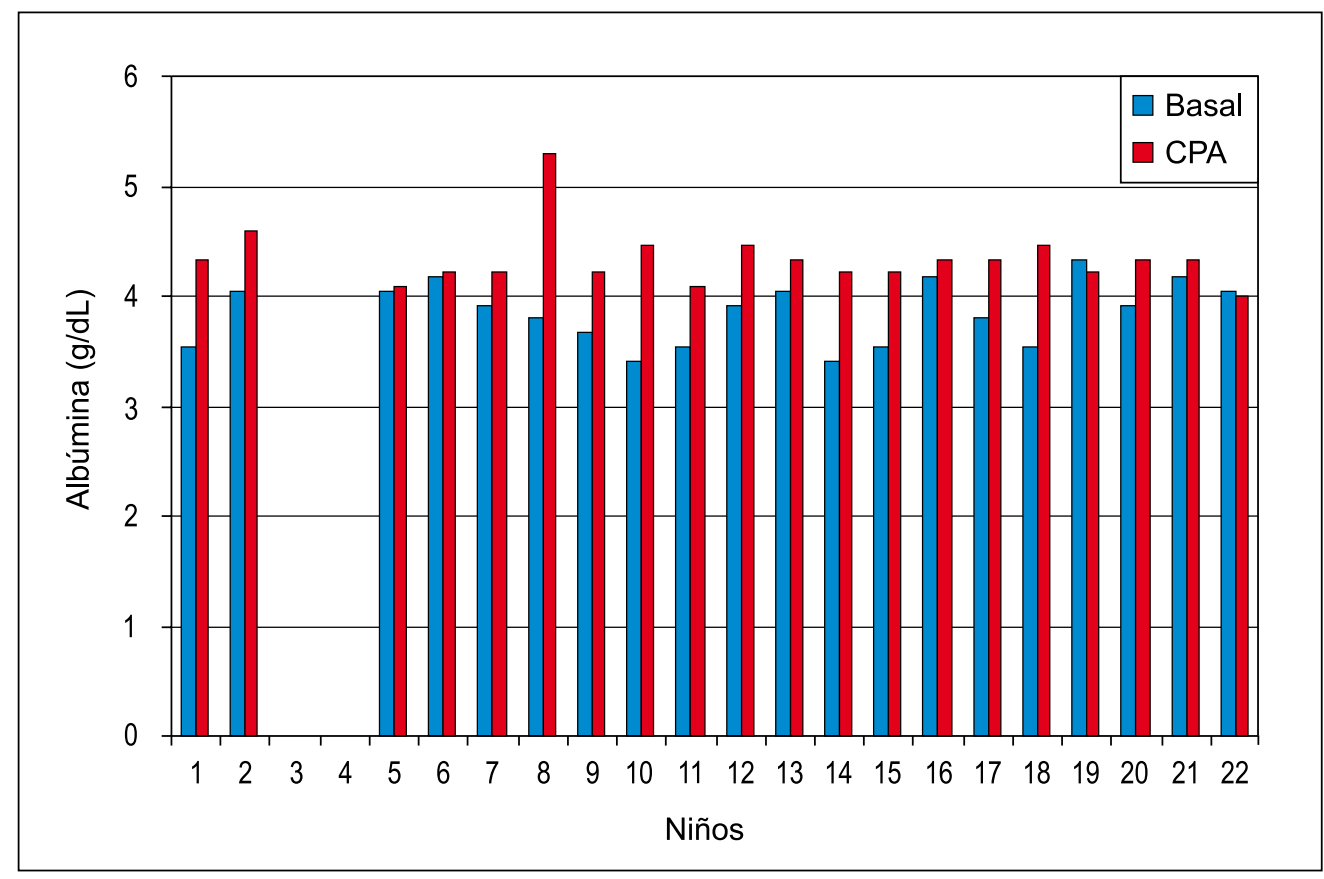

Figura 3. Valores de albúmina sérica antes (basales) y después de la intervención alimentaria con concentrado proteico obtenido de alfalfa (CPA). 


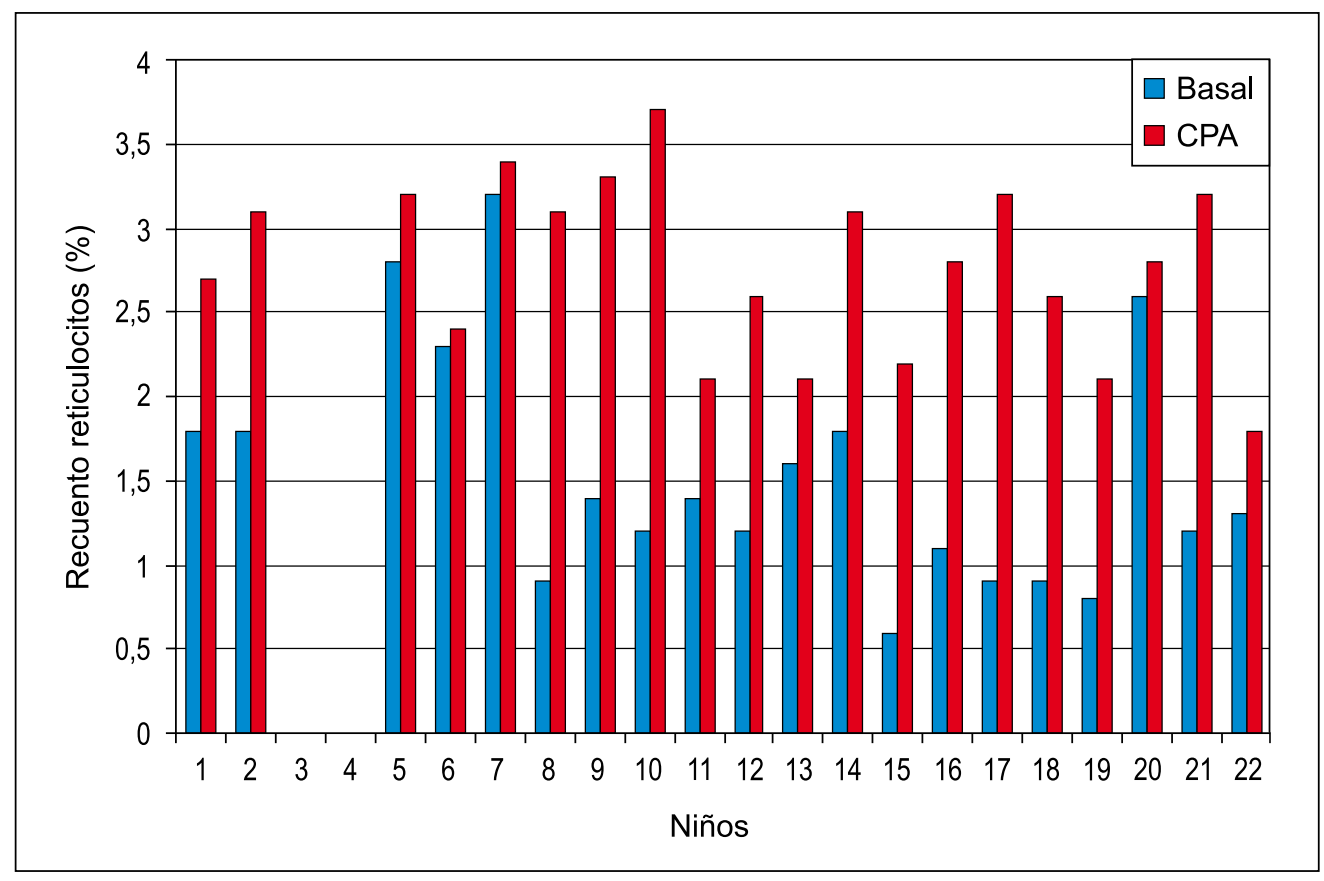

Figura 4. Valores de recuento de reticulocitos antes (basales) y después de la intervención alimentaria con concentrado proteico obtenido de alfalfa (CPA).

niveles de albúmina que muestran un incremento significativo $(p<0,05)$ después de la ingesta de $\mathrm{CPA}$, lo que indicaría una mejora en el estado nutricional de los niños intervenidos tras el consumo de CPA. Sería necesario determinar el contenido de aminoácidos esenciales presentes en el CPA y evaluar su aporte nutricional en la mejora de la desnutrición infantil.

En conclusión, el consumo diario de CPA, como suplemento alimenticio, por un período de 25 días, estimuló la eritropoyesis, debido a que incrementó los valores de recuento de reticulocitos y así mismo aumentó significativamente los niveles de albúmina en niños menores de 6 años con desnutrición crónica, que residían por encima de los $3000 \mathrm{~m}$ de altitud.

\section{AGRADECIMIENTOS}

Al Consejo Superior de Investigaciones de la UNMSM, por el financiamiento al estudio, durante el año 2008. Al Director Melchor Ortiz y a la profesora Zonia Alejandrina Condori Quillatupa, del Centro de Educación Inicial № 460, La Colpa, del distrito de Daniel Hernández, Pampas, Huancavelica, por las facilidades prestadas para la realización de este estudio.

\section{REFERENCIAS BIBLIOGRÁFICAS}

1. Instituto Nacional de Estadística e Informática. Encuesta Demográfica y de Salud. ENDES 2000. Lima: INEI; 2001.

2. Instituto Nacional de Estadística e Informática. Endes Continua 2004-2005. Informe Preliminar. Lima: INEl; 2006.

3. Gobierno Regional de Huancavelica. Plan Estratégico de Desarrollo Regional Concertado y Participativo de Huancavelica 2005 - 2015. Huancavelica: Gobierno Regional de Huancavelica; 2003.

4. Shah FH, Salam Sheikh A, Farrukh N, Rasool A. A comparison of leaf protein concentrate fortified dishes and milk as supplements for children with nutritionally inadequate diets. Plan Foods in Human Nutrition. 2003;30:245-58.

Tabla 4. Valores promedios de la evaluación bioquímica y hematológica obtenido en los niños seleccionados, después de 25 días de ingesta de concentrado proteico obtenido de alfalfa (CPA).

\begin{tabular}{lcccccc}
\hline & $\begin{array}{c}\text { Hemoglobina } \\
(\mathrm{g} / \mathrm{dL})\end{array}$ & $\begin{array}{c}\text { Hematocrito } \\
(\%)\end{array}$ & $\begin{array}{c}\text { Recuento de } \\
\text { reticulocitos } \\
(\%)\end{array}$ & $\begin{array}{c}\text { Proteínas } \\
\text { totales } \\
(\mathrm{g} / \mathrm{dL})\end{array}$ & $\begin{array}{c}\text { Albúmina } \\
(\mathrm{g} / \mathrm{dL})\end{array}$ & $\begin{array}{c}\text { Globulina } \\
(\mathrm{g} / \mathrm{dL})\end{array}$ \\
\hline Media & 13,48 & 38,6 & 2,78 & 7,42 & 4,34 & 3,09 \\
$\mathrm{DE}$ & 0,593 & 1,83 & 0,53 & 0,42 & 0,27 & 0,47 \\
$\mathrm{RR}{ }^{*}$ & 15 a 18 & $\mathrm{NR}$ & 2 a 5 & 7,5 a 9,0 & 4,5 a 6,5 & $\mathrm{NR}$ \\
\hline
\end{tabular}

DE = Desviación estándar.

$\mathrm{RR}^{*}=$ Rango referencial en niños de 2 a 6 años que habitaban en lugares por encima de $3000 \mathrm{msnm}$, utilizados por el Laboratorio de Bioquímica y Hematología de la Maternidad de Lima.

$\mathrm{NR}=$ No reportado.
5. Dillon JC, De Mathan 0. Les extraits protéiques de feuilles, une source de nutriments pour les enfants malnutrits. Cah Nutr Diet. 1998;33:379-83.

6. Asociación Soya de Nicaragua. Barrio Ariel Darce: SOYNICA; c2005 [citado el 25 junio de 2009]. Disponible en: http://www.soynica.org.ni

7. Cordero A, Oriondo R, Agüero Y, Soberon MM. Caracterización y valor biológico en ratas desnutridas, del extracto foliar seco obtenido de alfalfa Medicago sativa cultivada en el Perú [resumen]. An Fac med. 2006;67 Supl 1:s20.

8. Cordero A, Oriondo R, Soberon MM, Ramos R. Evaluación de la toxicidad hepática del extracto foliar de alfalfa Medicago sativa consumido como fuente proteica por ratas desnutridas [resumen]. An Fac med. 2007;68 Supl 1:s31.

9. Aste-Salazar H, Krumdieck CL. Diferenciación de hemoglobinas humanas en las grandes alturas. 
1. Hemoglobina fetal en recién nacidos y adultos. Ginecol Obstet (Perú). 1971;17:79-102.

10. Pajuelo J, Villanueva ME, Chávez J. La desnutrición crónica, el sobrepeso y la obesidad en niños de áreas rurales del Perú. An Fac med. 2000;61:201-6.

11. Cereceda M. Dietética. De la teoría a la práctica. Alimentación en las diferentes etapas de la vida. Lima: Fondo Editorial UNMSM; 2008.

12. lliquin M. Prevalencia de anemia en mujeres gestantes peruanas en edad fértil, según ajustes de hemoglobina por altitud. Tesis para optar el título de Médico Cirujano. Universidad Peruana Cayetano Heredia. Lima, Perú. 2000.

13. Yip R. Significance of an abnormally low or high hemoglobin concentration during pregnancy: special consideration of iron nutrition. Am J Clin Nutr. 2000;72(Suppl):272S-9S.
14. Dallman P, Siimes M. Iron deficiency in infancy and childhood. Report of the International Nutritional Anemia Consultative Group (INACG). Washington, DC: Library of Congress; 1985.

15. Sherriff A, Emond A, Bell JC, Golding J; ALSPAC Study Team. Should infants be screened for anaemia? A prospective study investigating the relation between haemoglobin at 8,12 , and 18 months and development at 18 months. Arch Dis Child. 2001;84:480-5.

16. González GF, Tapia V. Hemoglobina, hematocrito y adaptación a la altura. Su relación con los cambios hormonales y el periodo de residencia multigeneracional. Revista Med. 2007;15:80-93.

17. Gonzales GF, Gófiez C, Villena A, Guerra-García R. Hematología del nativo de altura. Hematocrito en niños de diferentes altitudes del Perú. Acta Andina. 1992;1:89-91.
18. Gofiez C, Donayre M, Villena A, Gonzales GF. Hematocrit levels in children at sea level and at high altitude: effect of adrenal androgens. Hum Biol. 1993;65:49-57.

Manuscrito recibido el 3 de agosto de 2009 y aceptado para publicación el 4 de setiembre de 2009.

\author{
Correspondencia: \\ María M. Soberón Lozano \\ Centro de Investigación Bioquímica y Nutrición \\ Facultad de Medicina, UNMSM \\ Av. Grau 750. Lima 1, Perú \\ Correo-e: msoberonl@hotmail.com
}

